\title{
The Gamma Factory path to high-luminosity LHC with isoscalar beams
}

\author{
M.W. Krasny, ${ }^{a, b, *}$ A. Petrenko ${ }^{c, b}$ and W. Płaczek ${ }^{d}$ \\ ${ }^{a}$ LPNHE, Sorbonne Université, Université de Paris, CNRS/IN2P3, \\ Tour 33, RdC, 4, pl. Jussieu, 75005 Paris, France \\ ${ }^{b}$ CERN, \\ Esplanade des Particules 1, 1211 Geneva 23, Switzerland \\ ${ }^{c}$ Budker Institute of Nuclear Physics, \\ Prospekt Akademika Lavrent'yeva 11, Novosibirsk, Russia \\ ${ }^{d}$ Institute of Applied Computer Science, Jagiellonian University, \\ ul. Łojasiewicza 11, 30-348 Krakow, Poland \\ E-mail: mieczyslaw.witold.krasny@cern.ch, alexey.petrenko@cern.ch, \\ wieslaw.placzek@uj.edu.pl
}

There are two main ways to increase LHC luminosity without upgrading CERN injectors: (1) modification of beam-collision optics and (2) reduction of beam transverse emittance. The former is followed in the ongoing high-luminosity upgrade of the LHC (HL-LHC), while the latter, applicable only to ion beams, is described in this contribution. The reduction of the beam emittance can be achieved by employing a laser-cooling technique to bunches of partially stripped ions at the SPS flat-top energy. In the case of the isoscalar calcium beams fulfilling the present beam-operation constrains, the transverse beam emittance can be reduced in this way by a factor of 5 during the 8 second long cooling phase. This would allow to reach the nucleon-nucleon luminosity $L_{N N}=4.2 \times 10^{34} \mathrm{~s}^{-1} \mathrm{~cm}^{-2}$, which is comparable to the levelled luminosity for the HL-LHC proton-proton collisions, but with reduced pile-up background. The calcium-calcium collisions have several advantages over the proton-proton collisions for the electroweak physics, such as precision measurements of the $W$-boson mass and $\sin ^{2} \theta_{W}$, clean observation of the Higgs-boson decay into $b \bar{b}$ in its photoproduction channel or BSM phenomena. If this scheme is confirmed by the future Gamma Factory proof-of-principle experiment, it could be implemented at CERN with minor infrastructure investments. This contribution is based on Ref. [1].

40th International Conference on High Energy physics - ICHEP2020

July 28 - August 6, 2020

Prague, Czech Republic (virtual meeting)

\footnotetext{
${ }^{*}$ Speaker
} 


\section{Introduction}

The Large Hadron Collider (LHC) collides effectively partonic bunches which are a dynamical mixture of quarks, gluons and photons. They are confined in protons or stable nuclei (ions) which guarantee their stability over beam acceleration and storage in the CERN accelerator complex. These partonic bunch carriers are characterised by their atomic and mass numbers: $Z$ and $A$.

The proton beams have been used so far for the precision studies of the Standard Model (SM) electroweak (EW) interactions and searches for beyond the Standard Model (BSM) processes, while the ion beams have been dedicated mainly to studies of strong-interaction phenomena, in particular quark-gluon plasma. The main reasons for that are the following:

1. Maximisation of the collision energy of partons, determined by the maximal magnetic field of the LHC dipoles - the maximal energies of partons are at least by a factor of 2 higher for protons than for ions at the same beam-particle magnetic rigidity.

2. Minimisation of the multiplicity of background particles obscuring the detection and measurement of the EW SM and BSM partonic processes. These background particles are produced in strong-interactions mediated collisions of spectator partons which accompany partons taking part in the SM EW or BSM processes of interest. For the low-luminosity LHC, characterised by a negligible multi-collision pile-up, the number of parasitic collisions of spectator partons grows quickly with the mass number $A$ of colliding particles and is minimal for the proton-proton $(p p)$ collisions.

3. Maximisation of the partonic-collision luminosity to search for very rare BSM processes and to reduce statistical errors of the SM EW measurements. This is because the number of partons transported to the collision point at the LHC is significantly smaller for heavy nuclei than for protons. The main reasons for that are: achievable yields of beam particles from proton and ion sources, effects of space-charge and intra-beam scattering, and parasitic beam-burning processes lowering beam safety in superconducting rings in the case of high- $Z$ nuclei.

\section{Ioscalar-ion beams}

In Ref. [1] we argue that in the high-luminosity phase of the LHC run (HL-LHC) the above arguments against using the nucleus-nucleus $(A A)$ collisions for the studies of the SM EW and BSM phenomena can be circumvented by introducing a novel operation scheme of the ion beams with a laser-cooling method as a means of reducing their transverse emittance.

In order to find the optimal balance for the LHC research programmes based on the collisions of the partonic bunches in the $p p$ and $A A$ operation modes, one has to address the following questions:

- What are the advantages of nuclei for which the partonic composition of the $u$ and $d$ valencequark, gluons and photons can be tuned to values optimised by physics goals?

- What is the optimal beam-particle choice to maximise the rate of SM EW and BSM partonic collisions with respect to the beam-burning parasitic collisions?

- What is the optimal beam-particle choice to minimise the pile-up background for the LHC beams at the highest partonic luminosity? 
- Which partonic-bunch carriers can maximise the effective rate of photon-photon collisions?

- Which choice of the beam particles can give the most precise experimental control of the flavour-dependent fluxes and effective emittances of partons?

The analysis presented in Ref. [1] shows that the isoscalar nuclei $(Z=A / 2)$ have many advantages with respect to protons in the HL-LHC research programme, in particular for the precision measurements of the SM EW parameters and the study of the EW symmetry-breaking mechanism. This might be not surprising as, historically, the progress in understanding the weak interactions in the 1970s, 1980s and 1990s was made by studying lepton scattering on isoscalar nuclei rather than hydrogen targets: (1) iron $(\mathrm{Fe})^{1}$ in the CDHS experiment at CERN [2], CCFR experiment at FNAL [3], NuTeV experiment at FNAL [4], E140 experiment at SLAC [5]; and (2) calcium (Ca) in the CHARM experiment at CERN [6]. There was also a proposal for special runs with the deuterium beam at HERA (DESY) to resolve the light-flavour structure of a proton [7]. Finally, a fixed-target muon-deuterium scattering experiment at the SPS was proposed to support the LHC precision-measurement programme [8]. The main goal of the latter two initiatives was to reduce the interpretation ambiguities of the SM EW measurements at the LHC.

The isoscalar-ion beams have never been considered as a means for precision studies of the SM EW and BSM phenomena at the LHC. The most likely reason for this is that no scheme has hitherto been proposed that would allow to achieve the partonic luminosities in $A A$ collisions comparable to the ones in the $p p$ collisions.

In Ref. [1] we propose such a scheme. It can be summarised briefly in the following steps: (1) modify the electron-stripping of the CERN ion beams such that the ions can carry a small number of electrons in their atomic shells over the SPS acceleration cycle, (2) use atomic degrees of freedom of such partially striped ions for Doppler-cooling of the beams by laser photons at the top SPS energy in order to reduce their transverse emittance, (3) strip the remaining electrons in the SPS-to-LHC transfer line, (4) accelerate such low-emittance ion beams to the top LHC energies and bring them to collisions in interaction points (IPs).

\section{Laser-cooling of calcium beams}

The isoscalar calcium $(\mathrm{Ca})$ beam has been chosen for the actual implementation of the proposed scheme. It satisfies the main beam-operation constraints and maximises the physics potential in both the EW and BSM sectors, see Ref. [1] for details.

In Fig. 1 we show schematically the processes of laser-photon absorption (upper panel) and spontaneous photon emission (lower panel) by the partially stripped ion (PSI). The laser-photon wavelengths can be adjusted such that only PSIs with momenta above the central value in a given direction absorb such photons. Then, the spontaneous emission of photons in a random direction results in reduction of the momentum spread of the PSI beam along the laser-photon direction. This phenomenon is very well-known in the atomic physics as the Doppler cooling.

The longitudinal laser-cooling for low-energy and low-intensity atomic beams in storage rings was demonstrated already in the early 1990s [9, 10]. In Fig. 2 we present the results of our simulations for the longitudinal cooling of the $\mathrm{Ca}^{17+}$-ion beam at the SPS top energy using (1)

\footnotetext{
${ }^{1}$ With a small non-isoscalarity correction for Fe.
} 
Excited ion after the photon absorption:
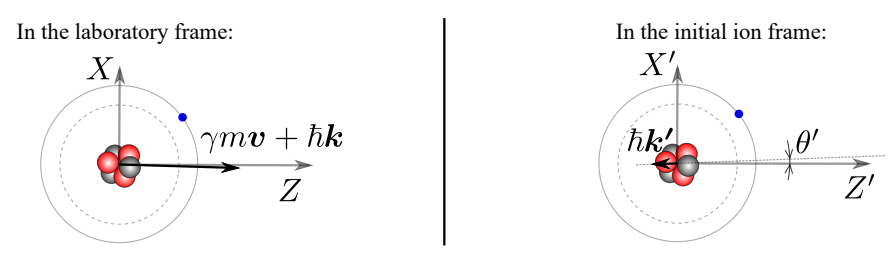

Ion after the photon emission:
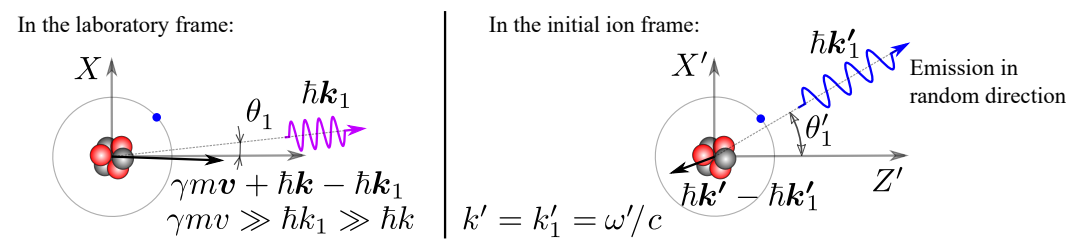

Figure 1: The excited PSI after the photon absorption (upper panel) and after the photon emission (lower panel).
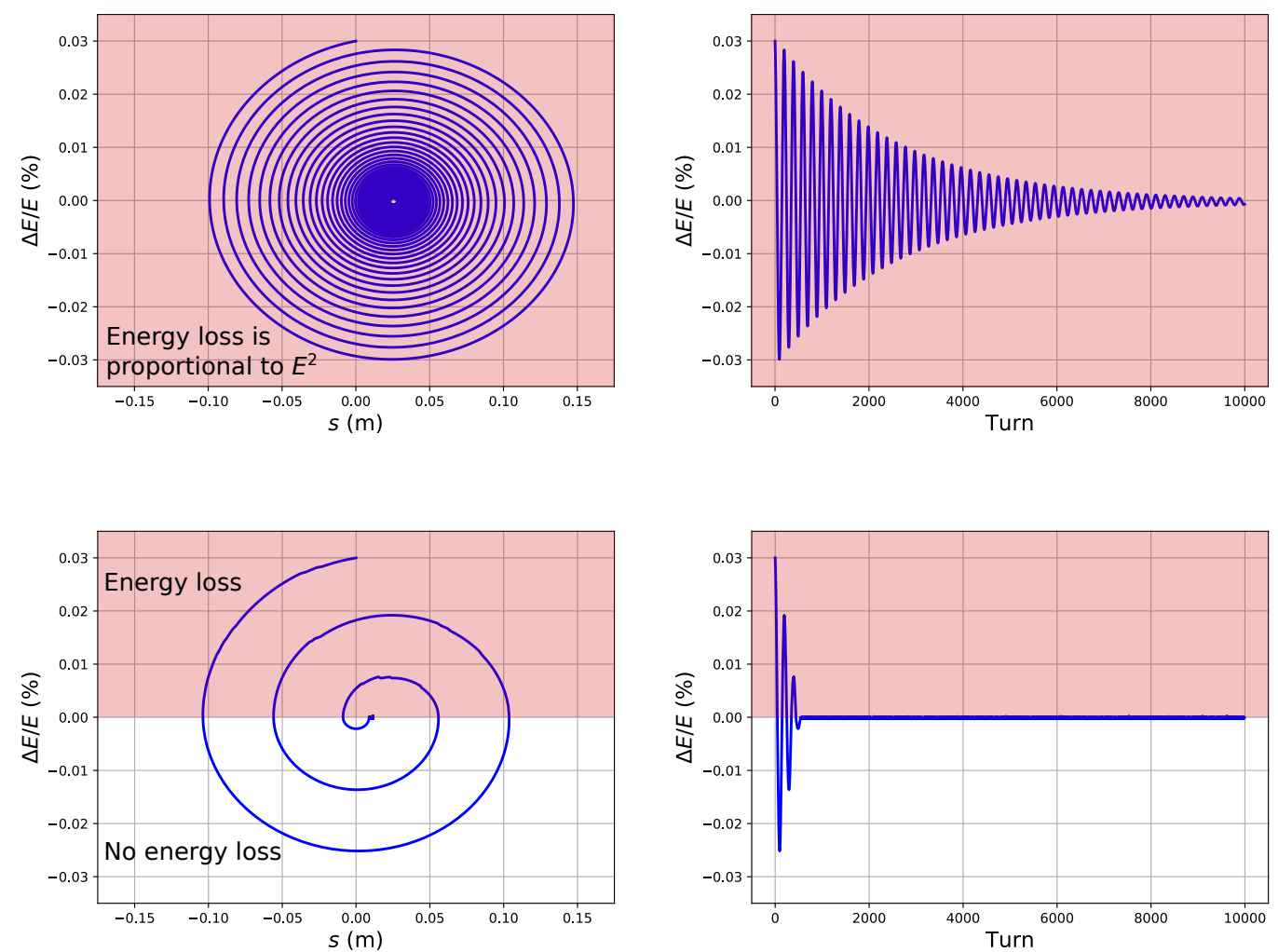

Figure 2: The evolution of the energy and the longitudinal position of the ion, relative to their central values, as a function of the turn number in the storage ring, for two regimes of the laser cooling: the upper plots show the broad-band laser cooling [15] using the laser frequency band which is large enough to excite all the ions, disrespectful of their energies; while the lower plots show the regime of fast cooling [16] using the laser frequency band which has a sharp cut-off, positioned such that the ion absorbs the laser photon only if the ion energy is above its central value. 
a broad-frequency-band laser - slow cooling (upper panel), and (2) a laser with a sharply cut-off frequency band - fast cooling (lower panel) [1]. In the SPS, due to limitations of the Ca-beam lifetime, the fast cooling technique must be applied.

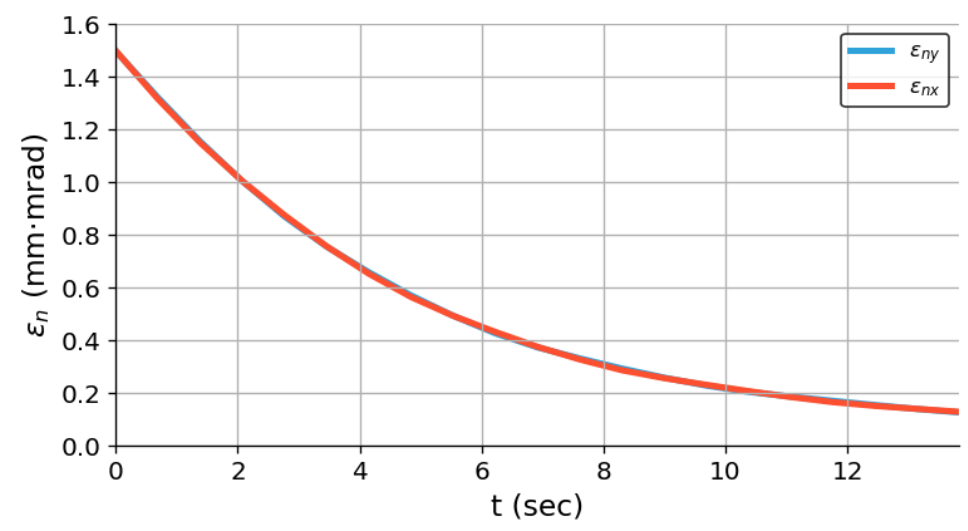

Figure 3: Transverse cooling speed: the time-evolution curves of the vertical and horizontal emittances overlap each other - they are equal when the betatron tunes are on the coupling resonance.

The transverse laser-cooling of low-energy and low-intensity atomic beams was studied in the late 1990s in Ref. [11]. In Fig. 3 we present the results of our simulations for the transverse $\mathrm{Ca}^{17+}$-ion beam cooling at the SPS [1]. As one can see, the reduction of the transverse beam emittance by a factor of 5 can be achieved within the 8 second long cooling phase. Assuming that the transverse emittance of such a beam is preserved over the phase of stacking of the SPS batches in the LHC and over its acceleration phase, the expected peak nucleon-nucleon (partonic) luminosity for the CaCa collisions is $L_{N N}=4.2 \times 10^{34} \mathrm{~s}^{-1} \mathrm{~cm}^{-2}$. This is higher than the levelled value for the high-luminosity $p p$ collisions with $50 \mathrm{~ns}$ bunch spacing and similar to that for the $25 \mathrm{~ns}$ mode.

The evaluation of various techniques of both the longitudinal and transverse cooling of PSI beams at ultra-relativistic energies is one of the important goals of the Proof-of-Principle (PoP) experiment [12] of the Gamma Factory initiative $[13,14]$ to be carried out at the SPS. If this experiment confirms the simulation results presented here and if such a beam-cooling scheme is implemented at CERN, it will open a way to high-luminosity operation of the LHC with nuclear beams. Adding such an operation mode to the LHC can extend the scope of physics studies and improve the precision reach of its scientific programme.

In Ref. [1] we argue that nuclear beams, and in particular the isoscalar ones, have numerous advantages with respect to the proton beams for the high-luminosity phase of the LHC operation. They allow to make the full use of the isospin symmetry of the strong interactions in constraining the flavour and momentum distributions of their partonic degrees of freedom. As a consequence, the analysis of the SM EW and BSM processes becomes insensitive to the limited precision of the LHC-external constraints, which will always remain essential for the interpretation of many $p p$ collision measurements. They can generate effective photon beams of unprecedented intensity, unreachable with the proton beams, opening the path to studies of the Higgs-boson production in photon-photon collisions, particularly its decay into $b \bar{b}$. Finally, they reduce the pile-up background in the high-luminosity phase of LHC operation while preserving the same partonic luminosity or, conversely, increase the HL-LHC partonic luminosity at a fixed soft-particle pile-up level. 


\section{Conclusions}

We have proposed a new operation scheme of nuclear beams at the LHC which includes a significant reduction of their transverse emittance by laser cooling. It opens the possibility to reach the partonic luminosities for the low- $Z$ nuclear beams which are comparable to that expected at the high-luminosity LHC phase of $p p$ collisions.

We have argued that colliding the isoscalar ion beams, such as the calcium beams, has numerous advantages over the $p p$ collisions for the LHC research programme, such as precision measurements of some Standard Model electroweak parameters (in particular $M_{W}$ and $\sin ^{2} \theta_{W}$ ), Higgs-boson studies (particularly its decay into $b \bar{b}$ ) and searches for beyond the Standard Model phenomena.

The proposed scheme can also be used at future high-energy hadron colliders, such as FCC-hh.

\section{References}

[1] M.W. Krasny, A. Petrenko and W. Płaczek, Prog. Part. Nucl. Phys. 114 (2020) 103792; [arXiv:2003.11407 [physics.acc-ph]].

[2] J.P. Berge et al., Z. Phys. C 49 (1991) 187.

[3] P.S. Auchincloss et al., Z. Phys. C 48 (1990) 411.

[4] M. Tzanov et al. [NuTeV Collaboration], Phys. Rev. D 74 (2006) 012008.

[5] P. Bosted et al., AIP Conf. Proc. 176 (1988) 508.

[6] J.V. Allaby et al. [CHARM Collaboration], Z. Phys. C 38 (1988) 403.

[7] M.W. Krasny, Electron-Deuteron scattering at HERA, in: Future Physics at HERA Proceedings of the DESY workshop 1995/96, Editors: G. Ingelman, A. De Roeck, R. Klanner.

[8] M.W. Krasny, F. Dydak, R. Voss, CERN-LHCC-2009-014 / LHCC-I-017, 17/09/2009.

[9] S. Schroder et al., Phys. Rev. Lett. 64 (1990) 2901; doi:10.1103/PhysRevLett.64.2901.

[10] J.S. Hangst, M. Kristensen, J.S. Nielsen, O. Poulsen, J.P. Schiffer and P. Shi, Phys. Rev. Lett. 67 (1991) 1238.

[11] I. Lauer et al., Phys. Rev. Lett. 81 (1998) 2052.

[12] M.W. Krasny et al. [Gamma Factory Study Group], Gamma Factory Proof-of-Principle Experiment, Letter of Intent, CERN-SPSC-2019-031/SPSC-I-253, 25/09/2019.

[13] M.W. Krasny, The Gamma Factory proposal for CERN, arXiv: 1511.07794 [hep-ex].

[14] M.W. Krasny [Gamma Factory Study Group], PoS EPS-HEP2017 (2017) 532.

[15] E.G. Bessonov and K.J. Kim, Phys. Rev. Lett. 76 (1996) 431.

[16] E.G. Bessonov and R.M. Feshchenko, Stimulated Radiation Cooling, Proceedings of RuPAC 2008, Zvenigorod, Russia. 